\title{
Ludoformer Lovecraft : Sunless Sea comme mise en monde du mythe de Cthulhu
}

Julien Bazile

\section{(2) OpenEdition \\ Journals}

Édition électronique

URL : http://journals.openedition.org/sdj/996

DOI : $10.4000 /$ sdj.996

ISSN : 2269-2657

Éditeur

Laboratoire EXPERICE - Centre de Recherche Interuniversitaire Expérience Ressources Culturelles Education

\section{Référence électronique}

Julien Bazile, «Ludoformer Lovecraft : Sunless Sea comme mise en monde du mythe de Cthulhu », Sciences du jeu [En ligne], 9 | 2018, mis en ligne le 06 juin 2018, consulté le 19 avril 2019. URL : http:// journals.openedition.org/sdj/996 ; DOI : 10.4000/sdj.996

Ce document a été généré automatiquement le 19 avril 2019

Tous droits réservés 


\title{
Ludoformer Lovecraft : Sunless Sea comme mise en monde du mythe de Cthulhu
}

\author{
Julien Bazile
}

1 L'influence du mythe de Cthulhu et plus généralement de l'œuvre de l'écrivain américain H.P. Lovecraft sur la culture populaire n'est plus à démontrer, tant sur le plan littéraire, cinématographique, musical que ludique. Qu'il s'agisse d'inspirations cachées ou de projets d'adaptation fidèles, on peut constater le nombre et la diversité des supports sur lesquels se propage l'univers lovecraftien. L'abondance des réflexions et des travaux sur Lovecraft menés par des amateurs éclairés comme par des universitaires ${ }^{1}$ en témoigne : la recherche ne se contente pas aujourd'hui d'étudier le corpus, vaste mais fini, de l'œuvre de cet auteur; elle cherche à en mesurer les influences, les transformations et les réécritures (Smith, 2011, p. 837).

2 Cette influence est notamment perçue au niveau de la réception, par une partie de la critique et des joueurs dans le cas du jeu Sunless Sea (Failbetter Games, 2015) ${ }^{2}$. Or, de l'aveu même de Lovecraft, toute tentative de matérialisation trop explicite ou trop littérale d'une horreur suggérée ne saurait que lui nuire ${ }^{3}$. Il s'agira de se demander dans le contexte de la présente réflexion quelles sont les spécificités du support vidéoludique convoquées pour cadrer l'expérience de jeu et tenir le pari de l'effroi et de l'irreprésentable. Comment s'établit l'analogie entre l'univers de Lovecraft et Sunless Sea?

\section{La ludoformation comme mise en monde ludique}

3 Pour comprendre comment une forme d'équivalence et de continuité sémantique peut se maintenir entre une source et son actualisation vidéoludique, nous chercherons à montrer que la transposition dont le mythe de Cthulhu fait l'objet ne repose pas sur une simple incorporation d'éléments explicitement issus de l'œuvre littéraire de Lovecraft. Le passage du récit littéraire au jeu vidéo peut être considéré comme une opération visant à fournir les moyens au joueur de faire l'expérience d'un univers fictionnel (Barnabé, 2014). 
Cette forme de remédiation ${ }^{4}$ du mythe relève donc de la mise en monde, de la simulation d'un cosmos régi par un certain nombre de lois.

Nous appellerons «ludoformation $»^{5}$ la transposition vers un support vidéoludique, le produit d'un ensemble de processus par lesquels une source est mise en jeu. Cette perspective cherche à souligner le passage d'un support ou d'un domaine sémiotique (un texte, un corpus de textes, d'images...) à un autre (le jeu numérique), au cours duquel se maintient une forme de continuité sémantique. Au-delà d'une influence unidirectionnelle, revendiquée ou non, l'enjeu est de remarquer comment l'œuvre transposée permet de jeter un éclairage nouveau sur la source, voire de participer à la définition et à l'extension de sa signification.

5 La ludoformation consiste ici, du point de vue de l'instance qui pose les cadres de l'expérience ludique, dans la mise en espace d'une narration spatialisée (Jenkins, 2002), c'est-à-dire son implémentation virtuelle basée sur des représentations graphiques et des mécaniques dont l'appréhension permet l'émergence de récits d'une certaine coloration, analogue à celle de l'œuvre originelle. La narration, comprise ici comme une «chaîne d'événements (parcours, séquence d'actions)» (Aarseth, 1997, p. 94), est directement tributaire de l'expérience de jeu. Elle émerge par l'intervention et la configuration directement opérées par le lecteur: elle n'existe, en l'attente de ce lecteur, que potentiellement.

La perspective du cybertexte permet de comprendre la ludoformation du mythe de Cthulhu dans Sunless Sea. Par cette catégorie de textes, Espen Aarseth désigne l'ensemble des phénomènes textuels dans lesquels « les différences fonctionnelles entre les parties mécaniques [...] jouent un rôle dans la détermination du processus esthétique » (Aarseth, 1997, p. 22). Il ne s'agit pas d'importer littéralement un ou plusieurs fils d'évènements advenus, mais de construire un système doté de propriétés spécifiques données à l'appréhension du joueur au moyen d'une jouabilité définie.

6 La perspective que nous proposons est celle de la ludoformation du « mythe » de Cthulhu dans l'œuvre de Lovecraft. Dès le départ, ce «mythe » est conçu par Lovecraft comme un cadre dans lequel se déroule l'action de plusieurs de ses histoires. Il est appelé dès l'origine à être poursuivi et étendu, plus qu'à demeurer un panthéon comptant une liste finie de divinités (Price, 1990). Il est une matrice à partir de laquelle émerge la nouvelle L'Appel de Cthulhu (H.P. Lovecraft, 1928). C'est au regard de cette matrice originelle que sera abordé le jeu Sunless Sea, lequel constitue un bon exemple pour étudier la façon dont une narration peut se déployer dans un jeu vidéo, particulièrement en référence à une œuvre préexistante.

7 Le «mythe de Cthulhu » fait référence à une partie de l'œuvre de Lovecraft, comprise généralement entre 1928 (la publication de L'Appel de Cthulhu dans la revue Weird Tales) et celle de la nouvelle Le Cauchemar d'Innsmouth en 1936. Il renvoie à un univers de sciencefiction/fantastique qui met en scène une série de personnages confrontés aux mystères d'un monde qu'un panthéon de dieux extra-terrestres, les Grands Anciens, tente de contrôler (Harms, 2008, p. 7-8). À la suite de Lovecraft, ce «mythe » est repris et étendu par des continuateurs, comme August Derleth (Bloch, 1987, p. 8). Ledit univers constitue un monde fictionnel qui fournit d'ores et déjà aux auteurs qui l'investissent un certain nombre de règles canoniques à respecter, mais dans le cadre desquelles ce monde peut être étendu. 
8 En tant que matrice, le mythe de Cthulhu peut être rapproché d'un architexte au sens de Genette c'est-à-dire comme modèle général de production de textes qui fait que ce dernier peut être mis en relation avec une "taxinomie " générique, textuelle ou discursive (Genette, 1982, p. 12). Une simulation comme celle que constitue Sunless Sea peut être observée en comparaison avec un mythe en ce que ce dernier constitue luimême un récit décrivant le fonctionnement d'un monde, qui met en scène des lois ou des puissances naturelles personnifiées avec lesquelles des protagonistes interagissent (Bratosin, 2007, p. 25). Effectivement, baptisé d'après le nom du Grand Ancien le plus célèbre, le «mythe de Cthulhu » désigne dès lors moins une cosmogonie, une collection de créatures mythologiques, qu'une cosmologie, une façon de représenter un univers et son fonctionnement.

Ludoformer un mythe peut consister dès lors conjointement à construire un monde et à penser la place d'un acteur dans ce monde. Il s'agit certes de représenter le monde fictionnel, car un jeu vidéo repose sur un régime de représentation graphique, audiovisuel et multimédia nécessaire pour rendre intelligible au joueur la proposition qui lui est faite par le jeu (Keogh, 2014, p. 7). C'est ce qui permet de justifier la présence d'emprunts littéraux au mythe lovecraftien (noms propres de personnages ou d'environnements décrits dans les œuvres littéraires). Mais plus encore, l'objectif est de le simuler, de dicter les lois qui régissent son fonctionnement.

Penser en termes de transposition d'un univers de fiction est d'autant plus opportun que, dans le cas de Lovecraft, la connaissance préalable de l'œuvre ne gêne pas une appréhension de son adaptation ludique. Une des forces de la mise en jeu de l'univers de Lovecraft réside certainement dans le fait que récits lovecraftiens et jeux vidéo ont pour ressort principal des jeux d'acteurs incertains, qui peuvent être identifiés comme les protagonistes d'un jeu (Hite, 2007) et/ou les personnages d'une œuvres littéraire (Berruti, 2004, p. 366). Dans d'autres cas, si l'œuvre originelle est reprise de manière trop littérale, les comportements du joueur peuvent s'en retrouver influencés (Dor, 2016, p. 2), ce qui nuit à l'expérience libre du jeu. Il s'agit donc de donner à (re)connaître Lovecraft dans un jeu, non pas seulement pour exprimer une filiation revendiquée par l'auteur du jeu, mais pour ouvrir des possibilités ludiques pour l'action du joueur, de faire en sorte que «l'histoire originale fourni[sse] un ensemble de représentations qui régiront les attentes vidéoludiques de l'utilisateur» (Genvo, 2005a).

11 Par la perspective de la ludoformation, on peut observer comment la conception de jeu établit une analogie entre une source et sa mise en jeu, en dotant le jeu et son univers de fiction de certaines règles, de qualités formelles qui contextualisent et cadrent l'action du joueur (Juul, 2005, p. 5), et font reposer celle-ci sur un certain nombre de " mécaniques », de méthodes employées par les joueurs pour interagir avec le système de jeu (Sicart, 2008).

12 Dérivé du jeu d'aventure narratif Echo Bazaar (sorti en 2009 sur navigateur), Sunless Sea met en scène un univers victorien qui emprunte au gothique, au fantastique et au steampunk. Le joueur incarne le capitaine d'un navire qui lui permet d'explorer une mer souterraine, la « Zee », afin de remplir un certain nombre d'objectifs qu'il se sera fixés en début de partie. Le jeu repose essentiellement sur la réalisation de quêtes liées à la collecte d'items, le dialogue avec les personnages et l'exploration de l'environnement marin, donnant lieu à des phases occasionnelles de combat naval. 
Dans un premier temps, nous verrons comment certains jeux vidéo lus dans la filiation de l'œuvre de Lovecraft peuvent être compris comme s'appuyant sur une continuité littérale, à la manière d'adaptations fonctionnelles. Cela nous amènera à distinguer la mise en monde à laquelle procède Sunless Sea, afin de proposer au joueur un cadre pour une expérience de jeu qui lui offre la possibilité de dérouler certaines séquences d'évènements. Nous verrons enfin comment mettre en jeu le mythe de Cthulhu consiste à convertir le récit d'évènements réalisés en un espace de configuration réglé par un système d'engagement. Elle permet de participer à l'élaboration d'une narration sur le mode de l'hypertexte ${ }^{6}$ et du cybertexte, réglée par les lois qui régissent le monde.

\section{Aux sources des jeux lovecraftiens : traductions formelles et traductions fonctionnelles}

14 Parmi les œuvres inspirées de l'œuvre de Lovecraft, on remarque que nombre d'entre elles prennent une forme de jeu. Outre de multiples jeux de cartes, le jeu de rôle sur table édité par Chaosium (Peterson, 1981) et ses multiples rééditions, ainsi que de jeux de plateaux (Horreur à Arkham, Launius, 1987), on constate que le jeu numérique tient une place notable dans cette ludographie. Depuis le jeu textuel The Lurking Horror (Infocom, 1987) jusqu'au dernier avatar de The Call of Cthulhu (Cyanide, 2017), trente années se sont écoulées et de nombreuses créations se sont vues attribuer un lien de parenté avec l'auteur de Providence.

Un bref tour d'horizon permet de constater l'emprise de Lovecraft sur le monde du jeu vidéo. Sur la plateforme de jeux Steam, les recherches sur les termes «lovecraft » ou " lovecraftian » renvoient entre vingt-cinq et trente résultats, quand la liste du site Lovecraft Ezine ne propose pas moins de soixante-seize jeux vidéo différents. ${ }^{7}$ Le célèbre hplovecraft.com dénombre quant à lui trente-et-un «Lovecraft-influenced» ou " Lovecraftian Digital Games ». ${ }^{8}$ En revanche, dans sa catégorie " Video games based on works by H. P. Lovecraft ", la version anglophone de Wikipedia recense seulement treize entrées. ${ }^{9}$

16 L'écart entre ces différents recensements montre que la définition de ce qui fait un jeu lovecaftien reste floue, ce qui complexifie l'attestation d'une filiation entre ces œuvres et la nouvelle originelle. Wikipedia ne prend en compte que les reprises littérales (un critère pouvant être la mention explicite d'un titre, tel The Call of (thulhu); d'autres au contraire comprennent plus largement l'univers et incluent alors des jeux présentant des thématiques analogues: la folie, la "connaissance interdite», la monstruosité. Ce qui permet alors de marquer un lien avec l'œuvre d'origine peut rendre accessoire toute référence explicite - visuelle, par le texte ou l'image - à celle-ci.

Dès lors, se demander si un jeu vidéo est une adaptation du mythe forgé par Lovecraft fait courir le risque de se heurter à deux écueils. Le premier serait d'aller observer dans une structure de jeu ce qui en elle fait explicitement et littéralement référence à l'univers de H.P. Lovecraft et de considérer ces éléments de référence comme nécessaires et suffisants pour faire de ce jeu un jeu lovecraftien. Adapter en jeu serait alors dans ce cas chercher une équivalence formelle, par le respect du format d'origine (Harvey, 2002, p. 46). Le souci de rendre ces éléments d'origine efficients dans un contexte nouveau, celui du medium videoludique, devient mineur. 
18 Le second écueil serait de ne rendre saillant dans un jeu, indépendamment de toute référence à l'œuvre d'origine, que certains des thèmes ou des concepts forts qu'elle contient. C'est ce que montre le jeu Eternal Darkness: Sanity's Requiem (Silicon Knights, 2002) qui met en scène la folie et la faillite de la perception en brouillant les codes de la jouabilité (gameplay). Hallucinations multiples, changements soudains de l'apparence du monde ou du protagoniste, perte de maitrise sur les actions de ce dernier : la capacité du joueur à maîtriser le jeu est altérée, afin de susciter un sentiment de désorientation analogue à celui du protagoniste.

Mais suffit-il de donner au joueur le sentiment d'être une créature insignifiante qui demeure engagée dans une démarche exploratoire (Øystein Slåtten, 2016, p. 69) pour attester d'une filiation lovecraftienne? Eternal Darkness opte pour une équivalence plus fonctionnelle que formelle, sur la construction d'un jeu aux mécaniques spécifiques qui proposent au joueur une «jouabilité lovecraftienne». Dans cette vision, serait alors «lovecraftien" un mode d'interaction particulier qui reposerait sur une perception limitée du joueur et son incapacité à comprendre le monde dans lequel il se déplace (Øystein Slåtten, 2016, p. 104-105) ou dans lequel ses actions n'auraient aucune portée sur le déroulement $\mathrm{du}$ jeu..$^{10}$ Or cette transposition, en prenant davantage en compte le support ludique de la remédiation, se réfère si peu à l'univers de fiction lovecraftien qu'elle ne peut prétendre maintenir spécifiquement une analogie avec le mythe de cthulhu.

Si des réécritures sont visiblement possibles, transposer cette œuvre dans la langue du jeu vidéo ne peut donc pas consister à transposer seulement des «éléments horrifiants fondamentaux » (Lovecraft, in Lacassin, 2010, p. 1054), ni à s'appuyer sur un registre de mécaniques de jeu générales propices au genre de l'horreur. Une manière pertinente d'approcher Sunless Sea comme traduction du mythe de Cthulhu peut être de comparer l'expérience narrative qu'il propose à celle des supports littéraires originels.

\section{Lovecraft mis en monde. Le jeu comme dialogue entre le navigateur et la carte}

21 Le jeu déploie une narrativité vidéoludique qui s'appuie sur une «narrativité statique, préétablie, scriptée » (des éléments posés par l'auteur, rappelant l'actualisation littéraire du mythe) ainsi que sur « une narrativité dynamique, produite par l'interaction du joueur avec le jeu » (Marti, 2014, p. 13). Dans le cas présent, l'adaptation du mythe de Cthulhu en jeu consiste à élaborer un parcours mettant en scène différents choix, à former des cadres de possibilités pour des types de parcours narratifs que l'on peut comprendre comme des "séquences d'actions" ou des "successions d'événements d'intérêt humains" (Bremond, 1966, p. 61) dont la coloration rappelle l'œuvre de Lovecraft. Cette traduction peut se comprendre comme un cybertexte, une structure qui permet la création de cette chaîne d'évènements qui ne se limite pas à un acte d'interprétation mais qui est précédée par une intervention directe du joueur (Aarseth, 1997, p. 114).

Il ne s'agit donc pas de considérer isolément chacun des éléments graphiques ou chacune des règles pour juger de leur inscription dans une filiation de l'univers de Lovecraft - ce qui fait dire à Raph Koster (2005, p. 172) qu'un « vecteur de force n'a pas d'agenda »mais de voir comment chaque élément, mis en cohérence dans l'objet créé, participe de la 
mise en monde. C'est cette dernière qui constitue la transposition, et c'est à l'aune de celle-ci que l'on peut établir une analogie plus directe avec le mythe.

\section{«Au milieu des noirs océans de l'infini ». Cartographie de l'angoisse}

« Nous vivons sur un îlot de placidité et d'ignorance au milieu des noirs océans de l'infini, et nous n'avons pas été conçus pour y entreprendre de longs voyages " (Lovecraft, 2013, p.13). Comment interpréter comme consigne de conception de jeu ces mots de Lovecraft?

L'auteur a manifesté son intérêt pour l'architecture, et notamment pour le pouvoir évocateur de son esthétique (Lovecraft, in Lacassin, 2010, p. 1127). Pour les commentateurs de Lovecraft, c'est une véritable architecture de l'horreur qui caractérise son œuvre (Houellebecq, 2010, p. 76-77). Le récit lovecraftien, comme la conception de niveau (level design) de Sunless Sea, évoque des constructions colossales d'un autre monde, et il est construit de façon à amener le lecteur à progresser au sein de celles-ci, et lui permettre par « éclairages successifs » de dérouler une narration.

Cette conception de l'horreur lovecraftienne rapproche le lecteur et le personnage en ce que chacun évolue à un rythme particulier (réglé par l'auteur, expérimenté par le joueur) dans la découverte des lieux et des actions. Le joueur progresse dans une partie de ce monde fictionnel (c'est-à-dire au cours d'une session de jeu, mais aussi dans un fragment $\mathrm{du}$ jeu), le héros lovecraftien mène son enquête, et les deux sont amenés de concert par l'auteur à faire face à une forme d'horreur dynamique. Celle-ci n'est alors pas une propriété intrinsèque des éléments du récit, mais elle est " rigoureusement matérielle " (Houellebecq, 2010, p. 36) et correspond davantage au régime de déplacement d'un agent dans un environnement. La conception de niveau de Sunless Sea peut être lue comme une traduction de cette progression vers l'horreur.

À la différence de nombre d'autres jeux, dans lesquels l'environnement ludique est un terrain de jeu dont le joueur doit s'assurer la maîtrise, il faut constater que Sunless Sea présente un espace d'une grande hostilité dont l'exploration est coûteuse pour le joueur. La gestion de l'espace ou déplacement au sein de celui-ci constitue à la fois un moyen de progresser et un marqueur qui sert à constater la progression (Chapman, 2016, p. 107). Cela se constate aussi bien dans les jeux de stratégie (la série Total War, Command and Conquer), les jeux de tir en vue subjective (Battlefield, Call of Duty), que dans les jeux de course (Gran Turismo, Burnout) ou les jeux de casse-tête (comme Tetris). Les mondes vidéoludiques font souvent de leurs environnements de jeu des terrains de conflits et de conquête, des champs de bataille où s'étendent des empires, où des ressources sont exploitées. Si une structure de jeu n'est pas réductible à son environnement spatial, celuici peut présenter au joueur certaines propositions d'engagement avec le jeu, l'informer sur des actions possibles ou évoquer une ambiance propice à développer certains comportements. 
Figure 1

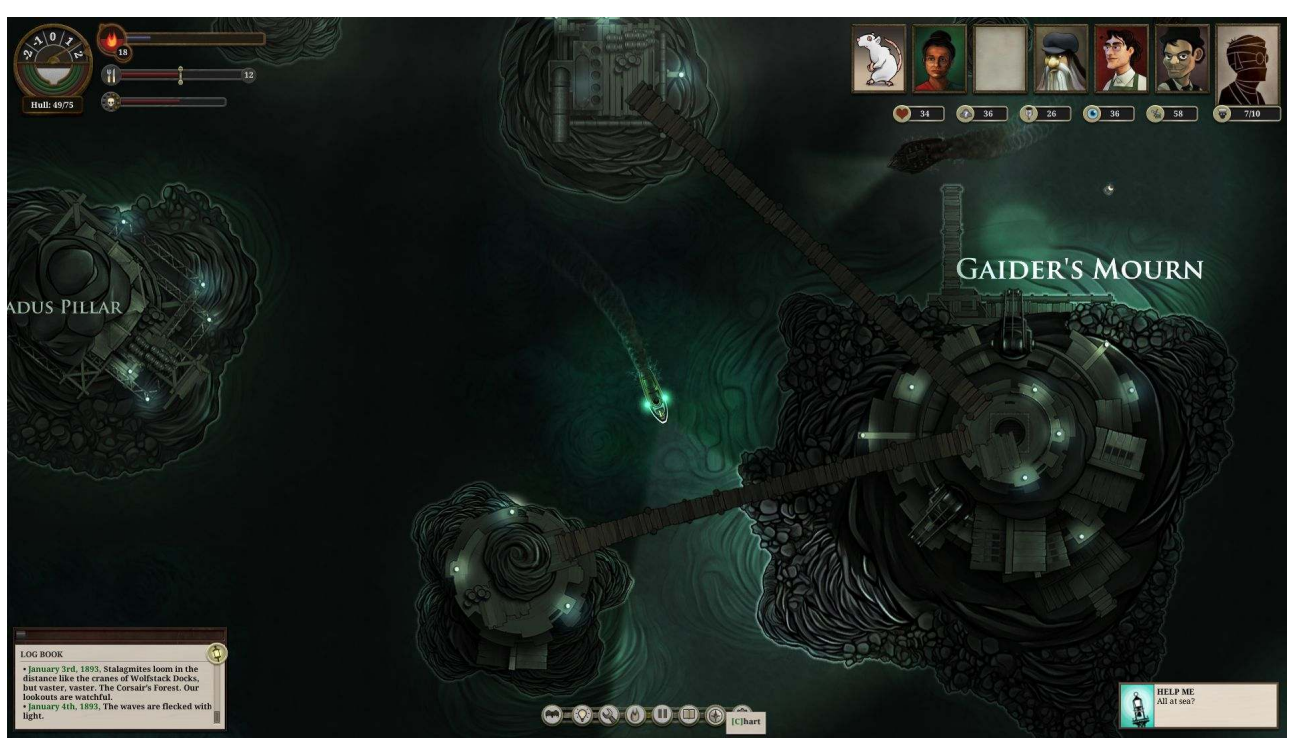

Mise en relief par le cadrage de la disproportion de l'architecture et de l'insignifiance du protagoniste

D'abord, la connaissance du monde ludique est médiée par une série d'indicateurs extradiégétiques (composant les éléments de la barre de statut ou de l'affichage tête haute HUD pour head-up display en anglais - destinés à informer le joueur sur sa progression. On constate dans Sunless Sea que ces jauges réclament la surveillance du joueur, puisqu'il doit modifier sa progression pour qu'elles ne se vident (nourriture et carburant) ou ne se remplissent pas complètement (terreur). À l'instar d'autres univers qui cherchent à modéliser un sentiment d'angoisse (Don't Starve, Klei Entertainment, 2013), Sunless Sea repose sur une mécanique de gestion de ressources qui entrave la progression du joueur.

La position du joueur sur la mer est matérialisée par un navire de petite taille, fiché au centre de l'écran, comme écrasé par l'univers dans lequel il évolue. Aucune carte de l' overworld (le monde fictionnel tel qu'il est accessible dans le jeu) n'est disponible tant que les différentes zones n'ont pas été explorées, obligeant le joueur à progresser en traversant ce «brouillard de guerre ${ }^{11}$ » qui entrave la lisibilité du monde. La progression dans cette mer vidéoludique s'effectue par le moyen d'une carte qui doit la représenter, qui pourrait la rendre intelligible mais qui ici la rend plus menaçante et impénétrable.

Cet environnement est le canevas à partir duquel le joueur prend la place du narrateur et construit une progression. Il faut remarquer qu'à la différence d'autres jeux de simulation maritime qui proposent des mers surpeuplées (citons Assassin's Creed IV : Black Fly, Ubisoft, 2013), Sunless Sea dépeint un univers d'un vide frappant. La possibilité de l'action frénétique et guerrière laisse place à la possibilité de la rencontre. L'accent est mis sur l'errance plus que sur l'exploration conquérante, sur l'attente, l'impression de présence, et amène le joueur lui-même à scruter fréquemment le monde à la recherche d'une direction. 
Figure 2

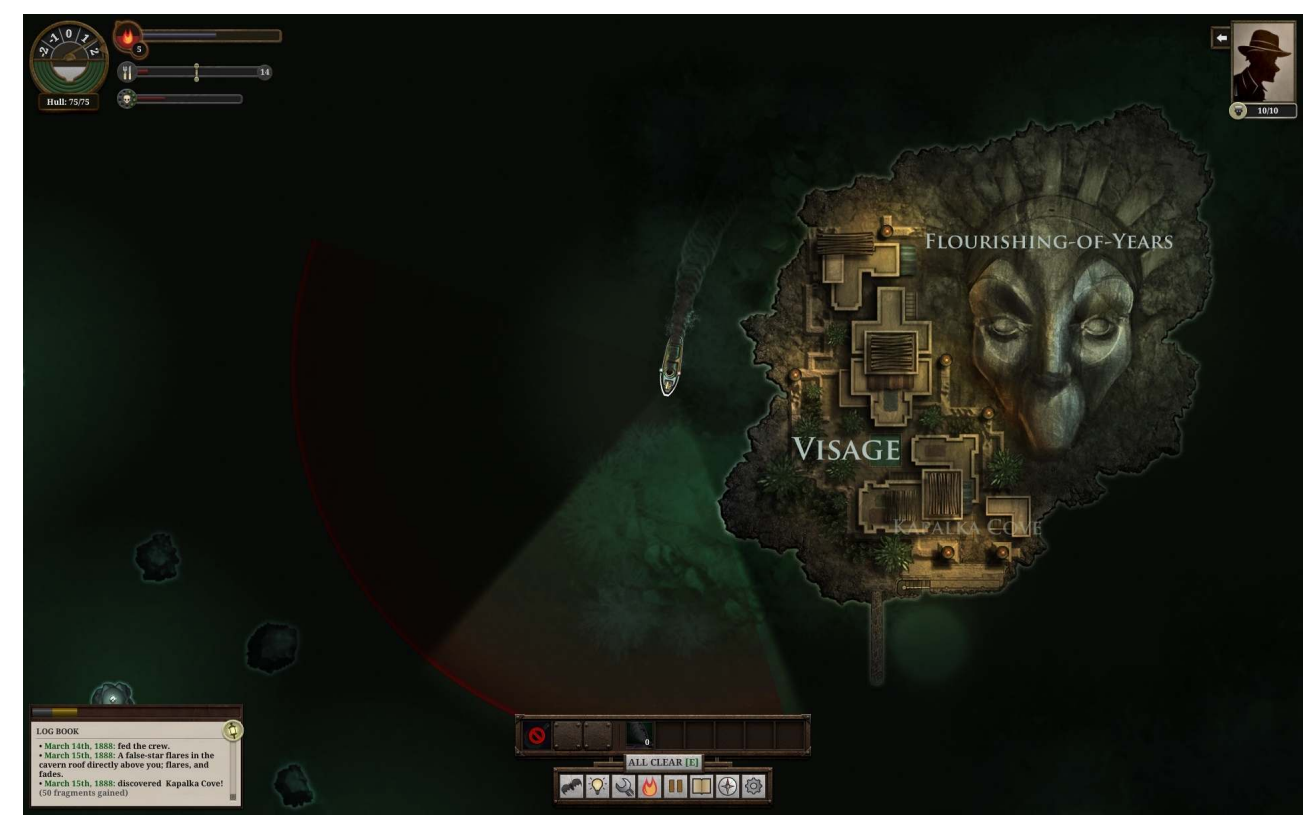

Un rivage au nom et à l'aspect cryptiques

Après son errance narrative, lorsqu'il rencontre enfin un élément (une créature, un navire, une île), les codes de représentation sont ceux de la cartographie maritime et des journaux de bord. La marche du temps est sanctionnée par l'apparition récurrente de dates dans un carnet de route ( $\log$ book) situé en bas de l'écran, accompagnées de relevés et d'observations sur le monde environnant (« Far off, very far off, someone screams... » [Loin, très loin, quelqu'un crie....], «The air trembles... » [L'air tremble], «Distant bells.» [Des cloches lointaines]). L'information est maigre, lacunaire et le joueur doit décider de la suite de sa progression dans un état permanent de pénurie. Les multiples sources d'informations sur la situation du joueur illustrent son rapport au monde.

31 Bien que cryptiques et indéchiffrables, le temps et l'espace demeurent dans l'oeuvre littéraire rationalisés en ce qu'ils font l'objet d'un décompte et d'un calcul (Houellebecq, 2010, p. 90-91). Dans le jeu, ils permettent au joueur de développer une stratégie afin de poursuivre sa progression. Les modalités de ce parcours participent d'une narration terrifiante qui rejoint celle de l'œuvre originelle. On le voit ici : le sentiment suscité par cette architecture ludique n'est pas une horreur aiguë et épidermique, mais bien davantage une angoisse (entendue à travers sa racine latine angŭsť̃a comme "resserrement»), causée dans le jeu comme dans l'œuvre par une "disproportion d'échelle» (Houellebecq, 2010, p. 97).

La condition du joueur, ici dans une position de narrateur, recoupe encore celle du protagoniste. Le monde mis en jeu est donné à voir comme au travers d'un écran radar, et constitue une vue du monde rationalisée et savante qui pourrait être celle des protagonistes lovecraftiens, "savanturiers » (Menegaldo, 2002, p. 181) qui progressent dans un monde qu'ils tentent de déchiffrer pour le rendre moins hostile. Les diverses entités, biologiques, insulaires - parfois les deux à la fois - qui peuplent le jeu ne se montrent pas forcément plus compréhensibles une fois rencontrées. Qu'il s'agisse de noms d'îles, de créatures, de machines ou de personnages, l'usage de noms curieux ou monstrueux rappelle celui des tératonymes lovecraftiens (Robinson, 2010, p. 129). Ils 
permettent d'observer un exemple criant d'objet ludoformé : la forme de l'objet est en lien avec sa fonction, la règle qui y est attachée, à l'image du monstrueux Cthulhu, dont le nom impossible à prononcer fait écho à une créature impossible à concevoir. L'analogie se trouve ici encore dans la jouabilité , dans la façon qu'a le joueur d'interagir avec l'univers du jeu.

L'un des avatars les plus significatifs de ce monde indéchiffrable se retrouve également dans la façon dont l'environnement est généré par le programme. À chaque nouvelle partie, l'emplacement des différentes îles est implémenté de manière quasi-aléatoire par génération procédurale. D'une partie à l'autre, chaque île se trouve toujours dans une certaine zone de la carte, mais située différemment par rapport à ses voisines. Cette organisation de l'espace et des éléments de sens au sein de cet espace tend à rattacher un peu plus la narration de Sunless Sea à celle qui peut être implémentée dans un cybertexte. Il s'agit de prévoir des éléments cachés, non forcément visibles mais conçus par l'auteur (les entités rencontrées sur la mer), dont l'apparition est régie par des mécanismes de production contenus dans le code (la génération procédurale, la restriction de la visibilité de la navigation sur la carte), et actualisés par l'action d'un joueur, qui effectue un parcours linéaire (Aarseth, 1997, p.51). Ce sont véritablement ces trois éléments combinés qui fondent « le texte » lui-même.

Le procédé n'est pas nouveau, et certains jeux ont fait de cet aléatoire contrôlé leur force. Mais cette mécanique de jeu n'est pas porteuse du même sens selon le contexte dans lequel elle est mobilisée. Dans un rogue-like (d'après le jeu Rogue, Toy et Wichman, 1980 ; famille de jeux vidéo dont l'unes des caractéristiques est la génération aléatoire de son univers au début de chaque partie) il est la garantie d'un renouvellement des scènes de combat, une promesse de challenge qui s'offre de manière perpétuelle au joueur. Dans un jeu d'exploration fortement narratif, il est davantage synonyme de perdition, de progrès cyclique ou d'incertitude.

Le procédé de transposition qui pose Sunless Sea au plus près du mythe de Cthulhu se trouve certainement dans le rapport de Lovecraft à l'architecture et à son pouvoir évocateur. Sunless Sea se comprend dès lors comme un système qui déploie une "narration algorithmique ", à savoir " un système qui se caractérise par un ensemble de règles préétablies, et la possibilité d'y introduire des variables » (Favre, 2000, p. 54, cité par Genvo, 2002). La conception de niveau contient les mécanismes ludiques qui permettront au joueur de produire les événements qui constitueront l'histoire. Le joueur ne se contente donc pas uniquement de découvrir des événements préétablis, mais il les configure directement et devient acteur et narrateur de son voyage ; il est simultanément celui qui parcourt un monde fictionnel et produit un récit sur ce monde. 
Figure 3

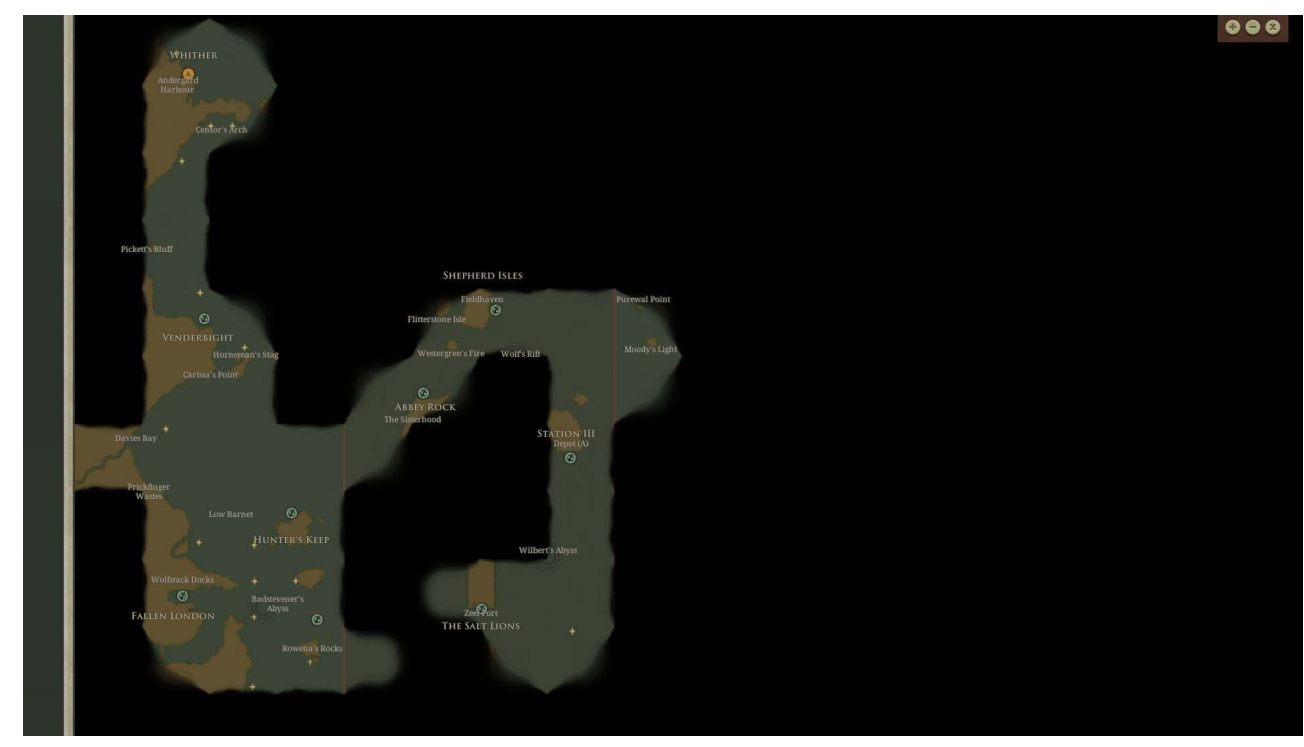

Les déplacements du joueur permettent de révéler la carte, qui représente le monde généré aléatoirement au début de chaque partie.

\section{« L'unification des connaissances dissociées »}

Les sciences, chacune poussant vers son propre but, nous ont jusqu'ici fait peu de mal; mais un jour viendra où l'unification des connaissances dissociées nous ouvrira de telles perspectives sur la réalité, et sur l'effrayante réalité qui est la nôtre, qu'ou bien cette révélation nous rendra fous, ou nous fera fuir cette mortelle clarté dans la mortelle sécurité d'un nouvel âge des ténèbres (Lovecraft, 2013 [1928], p. 13).

Sunless Sea est construit comme un jeu qui fait référence à la forme originelle dans laquelle il s'est déployé : il se déroule dans un univers ayant pour thèmes la recherche, la compréhension et même l'échange et la vente de récits et d'histoires. Les fragments d'histoires, les rumeurs, les légendes sont des ressources monnayables qui s'insèrent dans un marché. Explorer une terre lointaine est ainsi récompensé par le fait que le joueur puisse y recueillir un « rapport » qu'il lui sera possible de revendre à Fallen London, le port d'attache à l'extrême ouest d'où démarre chaque aventure.

Au cours de son voyage, le joueur est amené à explorer plusieurs fils narratifs. Le fait que ceux-ci soient enchâssés les uns dans les autres est un élément constitutif de la jouabilité : distincts et situés géographiquement, ils deviennent des ressources à atteindre. Ces « subnarrations " renvoient à une construction narrative propre à l'œuvre lovecraftienne, comme l'a mis en lumière S.T. Joshi (cité par Berruti, 2004, p. 369) : Sunless Sea démarre en proposant au joueur un objectif à long terme, qu'il devra atteindre au terme d'une succession de péripéties (de la même manière que l'intrigue principale suit une forme de strict chronology), au cours de la réalisation duquel il est informé sur des évènements antérieurs à son parcours (analogue aux flashbacks). Il est confronté à plusieurs pics de tension narrative lorsqu'il accomplit certaines tâches marquantes (double or multiple climax). Enfin, au cours de son parcours il est amené à lire et collecter des fragments d'histoire, qui établissent une parenthèse dans sa quête principale et ouvrent d'autres arc narratifs (narrative within narrative). 
Le jeu crée une expérience narrative singulière en alternant la navigation maritime avec des sessions de navigation dans des hypertextes, c'est-à-dire des textes dotés d'un système de renvois qui permettent au lecteur de se déplacer d'une section à l'autre du texte, selon les choix opérés. Le joueur (ou le lecteur) n'a qu'un rôle exploratoire dans ce type de texte statique, dont il n'est pas possible de configurer la forme (Aarseth, 1997, p. 62-64). Une part majeure de l'exploration du monde s'effectue au moyen d'un livre de bord présenté au joueur. Celui-ci agit comme une interface grâce à laquelle il peut commercer, suivre sa progression, acquérir des items et des ressources afin de développer une stratégie pour la suite de son exploration. Ce livre de bord remplit tour à tour le rôle de tableau de bord, permettant de concentrer des informations et des données sur la session de jeu, mais aussi de journal de bord, car c'est aussi à partir de lui que s'effectue une progression narrative hypertextuelle.

Alors que les jeux vidéo ont pu entretenir l'idée d'une contradiction entre séquences ludiques et séquences narratives du fait de la rupture que causaient les cinématiques (Solinski, 2017, p. 19), Sunless Sea propose une expérience d'exploration à la fois ludique et narrative. Chaque île ou chaque port visité offre au joueur la possibilité d'explorer un récit. La navigation dans ce fil narratif s'effectue sur le mode d'un hypertexte ludique, dans lequel le joueur est face au choix de certains possibles narratifs.

La navigation dans cet hypertexte repose en partie sur un calcul stratégique et témoigne de l'inscription du joueur dans un contexte de disponibilité ou de pénurie de ressources. Lorsqu'il est donné à entrevoir les conséquences des choix, tous ne sont plus présentés à égalité : certaines voies ouvrent sur des actions au pourcentage de réussite infinitésimal, ou demandent une consommation importante de ressources, tandis que d'autres promettent des risques plus faibles voire des gains substantiels. Toutefois, le calcul et la décision optimale dans l'incertitude ne sont pas les seuls à entrer en jeu, et le choix d'une ligne narrative demande aussi au joueur de se penser comme protagoniste au milieu d'un parcours : son choix sera aussi fonction des chemins narratifs qui auront été empruntés auparavant, notamment dans les phases d'explorations maritimes. Ces attentes narratives fondées sur un chemin parcouru rapprochent un peu plus Sunless Sea des livres-jeux, plus particulièrement des livres dont vous êtes le héros, dans lesquels un lecteur pouvait tomber sur un passage de plusieurs manières différentes, affectant sa compréhension du passage en question. 


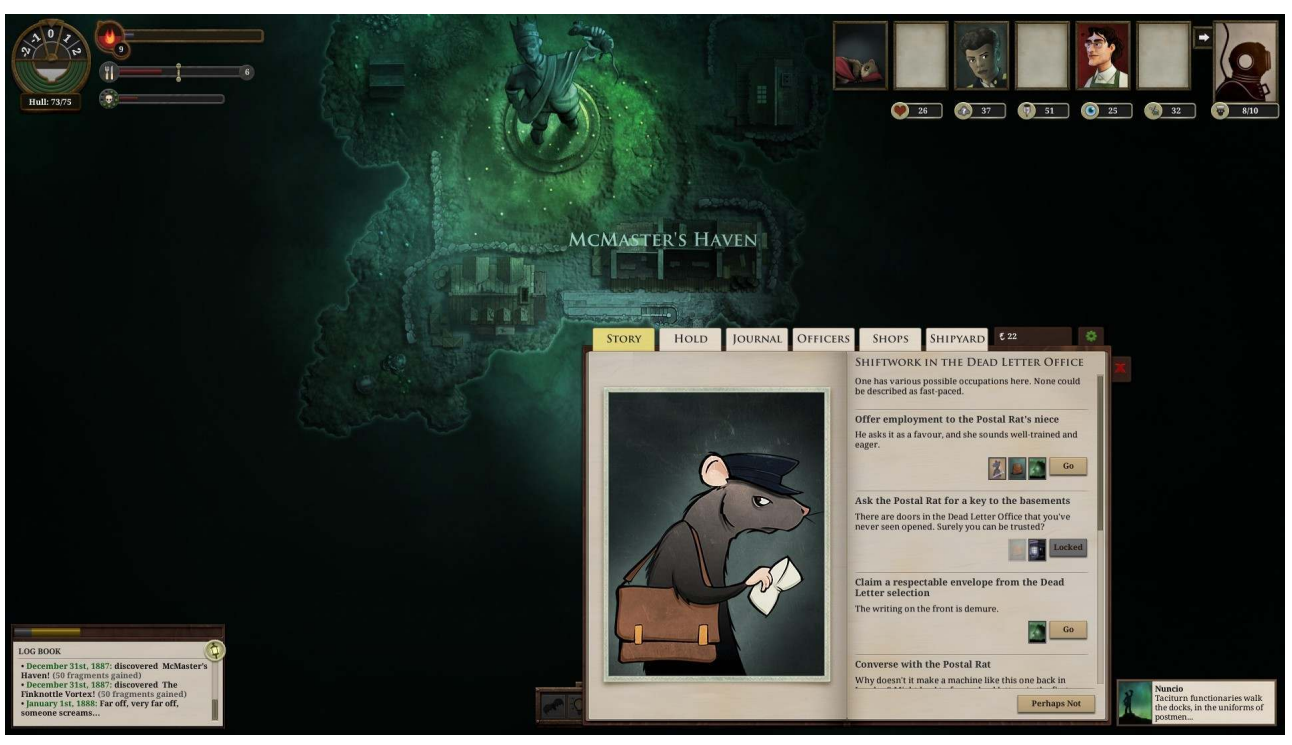

Une séquence de narration interactive à choix multiples. Les options sont parfois conditionnées par la possession de ressources ou d'items.

41 Le joueur-narrateur n'est cependant pas le seul décisionnaire du parcours narratif. On constate bel et bien en amont la présence d'un auteur qui restreint les possibilités narratives pour ensuite faire directement intervenir le joueur (Bouchardon, 2012, p. 19). L'auteur est présent mais ne décide pas de l'ordre d'apparition des évènements, de même que le concepteur de jeu (game designer) ne contrôle pas la partie qui sera jouée. Il s'agit de cadrer seulement : constituer une structure qui ne laisse pas le joueur se saisir d'un optimum, mener une stratégie unique qui permette de l'emporter aisément, par exemple en conditionnant la victoire au fait de faire se succéder de manière linéaire la liste des évènements prévus par le développeur. Le déséquilibre crée le jeu, et ce qui est saillant dans cette architecture narrative est bel et bien le choix offert au joueur.

Parler de « ligne» ou de « fil » narratif pose ici toutefois la question de l'existence d'une unité de narration sans Sunless Sea. A la différence d'une œuvre littéraire dont «l'intégrité [...] dépend de l'ordre de séquence de ses parties » (Ingarden, 1973, pp. 305-313), on ne peut distinguer dans le cas de Sunless Sea une telle stabilité linéaire. Pour autant, on ne peut pas en déduire que cette œuvre ne présente aucune unité sur le plan du récit. Si les évènements déclenchés par l'action du joueur peuvent apparaître comme agencés aléatoirement, ils ne le sont toutefois que selon certaines règles prohibant les combinaisons incohérentes. Cela empêche notamment l'apparition d'évènements qui nécessitent la réunion de conditions spécifiques (possession de ressources, avancement dans l'intrigue, sélection par le joueur), permet de respecter l'enchaînement logique des causes et des conséquences dans le cadre du récit et de s'assurer que la partie jouée par le joueur donne lieu à une séquence cohérente d'évènements successifs.

Il n'y a pas dans Sunless Sea d'unité de récit au sens de la théorie littéraire classique, si ce n'est celle qui réside dans ces règles de distribution des séquences évènementielles, qui cherchent à garantir une unité de stratégie et d'expérience pour le joueur. Le récit progresse par l'interaction entre joueur et jeu, et cette interaction permet de rendre compte de manière cohérente de la façon dont les évènements se succèdent. 

d'organiser sa partie pour l'atteindre. Certaines «ambitions» formulent des buts relativement explicites (" find your father's bones, and then retire » [trouvez les ossements de votre père, puis prenez voter retraite], « found your own private kingdom » [fondez votre propre royaume privé ]), tandis que d'autres sont plus cryptiques (« liberate the secret of immoratily »[libérez le secret de l'immortalité], « sail East, beyond the Zee » [naviguez vers l'Est, par-delà de la Zee]). Le joueur sélectionne lui-même cet objectif, et la possibilité lui est offerte de suivre les pas de l'aventurier qu'il a précédemment incarné. Les avatars que le joueur peut sélectionner ont la forme de silhouettes grisées, signe de leur caractère interchangeable, à l'image sans doute des personnages lovecraftiens dont on a pu dire qu'ils manquent de personnalité (Berruti, 2004, p. 376), ou n'ont pour «seule fonction réelle [que] de percevoir » (Houellebecq, 2010, p. 83). Sunless Sea se rapproche alors un peu plus du mythe que, par exemple, le jeu de rôle sur table édité par Chaosium, en ce que les nouvelles originelles reposent sur le principe que l'enquête soit résolue sans que l'horreur ne soit vaincue (Hite, 2007), et les tentatives de progresser dans la compréhension du monde se succèdent comme autant de parties jouées.

faire émerger des récits, la génération, la représentation et l'interactivité de ce monde sont gérées par un ensemble de règles que l'on peut qualifier de lovecraftiennes, rappel de ce thème du monde à l'histoire cyclique, de l'éternel réveil du péril (Menegaldo, 2002, p. 186) dans un monde horrifique qui survit au protagoniste. Du fait de la génération procédurale de la carte, les efforts d'un joueur consciencieux qui aurait cartographié le monde qu'il a parcouru lors de sa précédente partie se retrouvent presque réduits à néant. C'est un nouvel élément qui rattache Sunless Sea à la famile des cybertextes; leur parcours peut être couronné de succès ou donner lieu à un échec (Aarseth, 1997, p. 90-92). Par ailleurs, cette génération procédurale ne détruit pas toute forme d'unité dans le récit proposé par le jeu. De même qu'une œuvre cinématographique montée dans un ordre non prévu par son auteur ne constitue pas un "nouveau film ", chaque partie jouée n'est pas entièrement une production nouvelle, car «la séquence originale comme la séquence hérétique sont toutes deux basées sur le même potentiel matériel » (Aarseth, 2003, pp 763-765). Le récit implémenté dans le jeu vidéo consiste ici en l'ensemble des évènements et des règles régissant l'apparition et la succession de ceux-ci, et prend la forme d'un «langage limité » (Aarseth, 2003, p. 765) dont on peut 
connaître les parties constitutives mais pas la combinaison, produite par l'action du joueur.

Figure 5

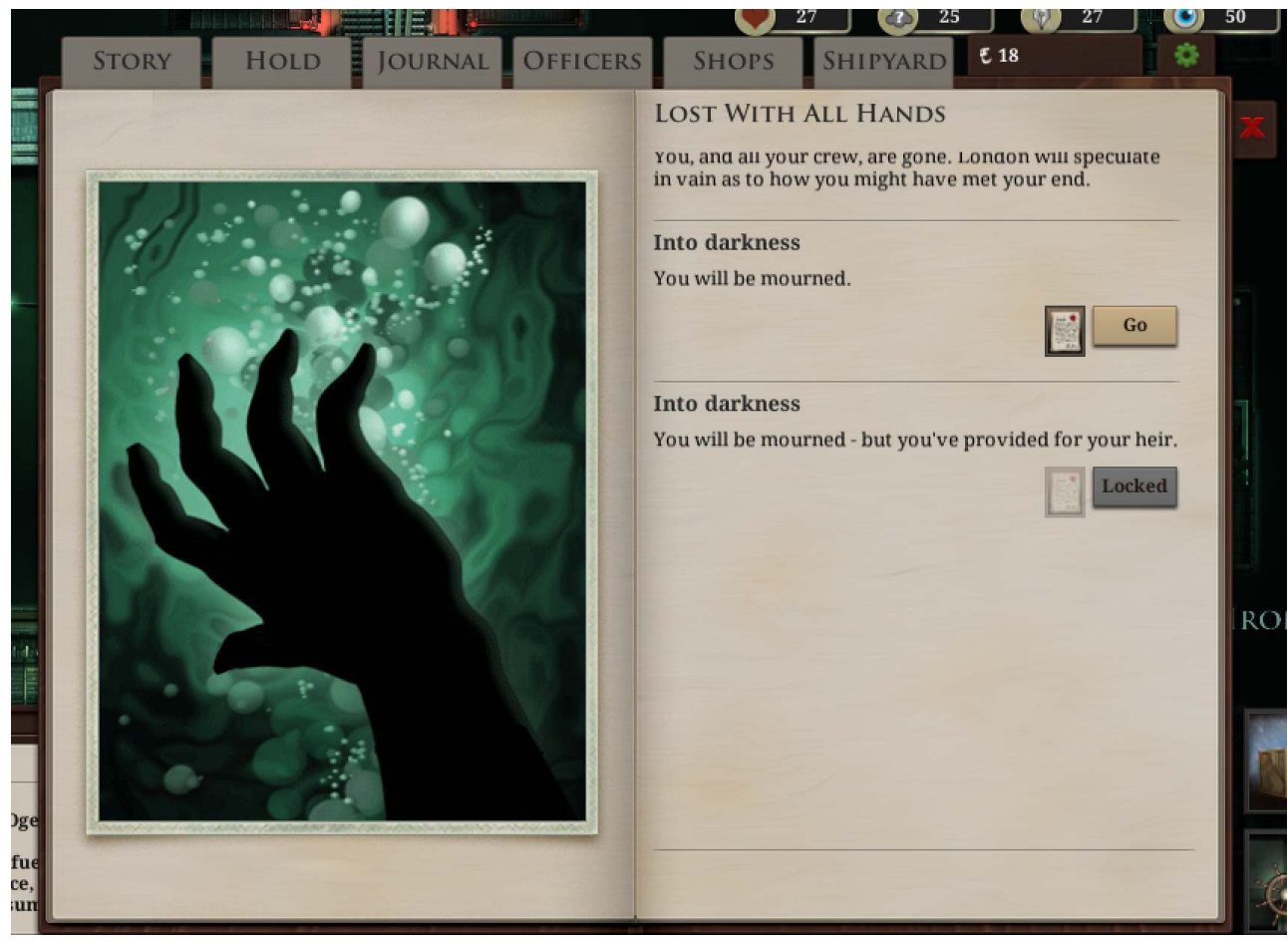

L'évènement sanctionnant l'échec (la mort du personnage joueur). II peut donner lieu à un transfert de ressources, de compétences ou d'items pour la partie suivante (ici, l'option est indisponible).

Les récits (d'horreur, de voyage, d'enquête) sont au cœur des mécaniques de jeu. Comme monnaie d'échange, ils motivent le voyage qu'effectue le joueur; comme ressources, ils le rendent possible; comme fenêtres sur le monde fictionnel, ils lui donnent du sens. Cette façon d'intégrer des éléments de narration à la structure de jeu tend à réduire la dissonance entre le déroulement d'une histoire implémentée et la pure configuration libre, et fait émerger une forme de narrative play. Selon cette conception, «la connexion entre les mécaniques au cœur du jeu et l'histoire qui s'y déploie peut se trouver dans les conditions de victoire, ainsi remporter une victoire revient à raconter la meilleure histoire » (Mitchell et McGee, 2009, p. 101). Réussir le jeu est ici partiellement conditionné par l'attention portée aux récits, en tant que ressources présentes dans le jeu; une attention que l'on peut qualifier de lovecraftienne en ce qu'il s'agit de partir à leur recherche, de les recueillir, d'en comprendre le sens et de s'appuyer sur eux pour progresser.

\section{Du monde ludique au monde narratif}

\section{L'oeuvre de Lovecraft comme genre vidéoludique?}

Pour certains, la source première d'un jeu, celle dont il faut prioritairement penser la remédiation, est à chercher dans son appartenance générique. Selon cette conception, une œuvre originale n'est qu'un paratexte ${ }^{12}$ par rapport au jeu. Ce dernier doit être 
compris essentiellement au travers de sa jouabilité (Randall, 2017, p. 173), lequel s'inscrit dans un genre vidéoludique défini. Contrairement à Cthulhu Saves The World (Zeboyd Games, 2011), qui est caractérisé par sa reprise des codes du jeu de rôle japonais (J-RPG), dont la distinction des phases d'exploration et de combat, ce ne sont pas de tels codes vidéoludiques qui permettent de saisir la spécificité de ce jeu.

La compréhension des jeux vidéo ne se limite pas à leur inscription dans un genre compris comme ensemble de mécaniques de jeu spécifiques. Ils fonctionnent selon un régime de remédiation complexe. Ils peuvent eux-mêmes agréger plusieurs formes médiatiques telles que le son, l'image animée, le texte (Flanagan, 2017, p. 441-448), si bien que leur caractère singulier réside dans une composition hybride de règles arbitraires et de contenus multimédia, formant une structure complexe non réductible à une seule de ses composantes (Keogh, 2014, p. 10). Mais si, prise isolément, chacune des sources de Sunless Seane peut fonder à elle seule une analogie convaincante avec le mythe, considérées ensemble elles constituent le matériau mobilisé pour construire un monde ludique, qui lui peut être lu par sa filiation avec le mythe de Cthulhu.

51 L'une des spécificités de Sunless Sea réside notamment dans le fait qu'il contient des règles inspirées de plusieurs genres. Ainsi, les mécaniques propres aux jeux de rôles classiques qu'il contient (les quêtes, la liberté d'exploration, les compétences à améliorer) poussent les joueurs à l'exploration et à l'expérimentation, alors que les éléments de rogue-like (la génération procédurale, la sauvegarde unique ou le système de mort permanente du personnage) punissent les joueurs pour leurs erreurs. Le "genre lovecraftien» dans lequel on peut inscrire ce jeu n'est pas une catégorie substantielle qui repose sur un ensemble de mécaniques définies, mais plutôt un ensemble multifactoriel qui porte la trace de ses filiations et de ses influences.

Inscrire un jeu dans la filiation d'un genre vidéoludique consiste souvent à chercher dans ce jeu une continuité en termes de mécaniques de jeu. Cependant, ce sont les jeux euxmêmes qui participent à la définition des genres (on a d'abord parlé de rogue-like, Grand Theft Auto-like, Doom-like, Myst-like, en référence aux œuvres qui ont popularisé les mécaniques sur lesquelles ces genres reposent). Un genre vidéoludique n'est pas toujours une catégorie stable issue d'une observation, mais plutôt une matrice empirique à partir de laquelle créer, un guide pour le développeur. De la même manière, inscrire un jeu dans la filiation d'une œuvre littéraire suppose de considérer l'œuvre comme une matrice, fournissant des consignes pour élaborer le cadre du jeu. S'appuyer sur les seules conventions génériques fait courir le risque de faire de l'œuvre originelle non pas un paratexte mais un prétexte pour recréer des jeux fondés sur une famille de mécaniques éprouvées. C'est pour cela qu'on a pu noter que la «rhétorique cthuléenne » de la terreur cosmique ("cosmic terror») s'oppose à la «rhétorique du contrôle» habituellement implémentée dans des jeux de tirs et de survie (Krzywinska, 2009, p. 278). Les jeux les plus efficients en tant que traductions de Lovecraft semblent être ceux qui parviennent à maintenir un "équilibre entre contenus lovecraftiens et ambiances lovecraftiennes » (Flanagan, 2017, p. 448).

\section{La machine et son opérateur. Sunless Sea comme cybertexte}

Un jeu vidéo doit nécessairement laisser une place de premier plan au joueur : il demande de s'affranchir du poids d'une instance auctoriale. Si la narration est littéralement implémentée et que le joueur ne fait que la dérouler, il n'y a plus de jeu. De même, 
retranscrire un monde ludique trop hostile constituerait un système qui empêcherait toute interaction. Un personnage fréquemment sidéré devant la terreur découverte (Krzywinska, 2009, p. 277) serait proprement injouable. Il en est de même d'un univers de fiction qui serait parfaitement indifférent à la présence du joueur ou qui lui serait inacessible. Dès lors, pour mettre en scène un monde lovecraftien qui laisse la place au joueur, le concept de cybertexte semble pertinent pour penser cette mise en monde.

Il est nécessaire de considérer la construction d'un texte, un assemblage d'éléments signifiants, qui fasse du joueur sinon «l'auteur du récit dans le jeu» (Marti, 2014, p. 11), du moins l'une des instances qui va occuper le rôle du narrateur. Il s'agit donc de conditionner l'émergence des récits à l'intervention du joueur. Aarseth le fait s'exprimer par la formule : «je veux que ce texte raconte mon histoire, l'histoire qui ne pourrait pas être sans moi » (Aarseth, 1997, p. 4)

Le cybertexte se comprend aussi parfaitement dans les coordonnées d'une architecture narrative (Jenkins, 2002). Tel que défini par Espen Aarseth : « il est un univers de jeu [...] il est possible de l'explorer, de s'y perdre et de découvrir des passages secrets dans ces textes, non de manière métaphorique, mais grâce aux structures topologiques de la machine textuelle» (Aarseth, 1997, p.7). Par ailleurs, il remarque que la forme vidéoludique se trouve être extrêmement liée à des questions d'organisation spatiale : il présente une forme "d'allégorie d'espace» (Aarseth, 2001, p. 154, 169), où les lois physiques et sociales qui régissent l'espace (réel ou fictif) sont déviées, remplacées par les règles du jeu.

Une traduction d'œuvre littéraire sur support vidéoludique doit alors prendre la forme d'un système qui soit une invitation à l'action (Genvo, 2005b). Pour que la cartographie du monde de terreur cosmique de Lovecraft puisse fonctionner, il est nécessaire de penser l'agentivité et la curiosité du protagoniste qui doit y évoluer. La conscience aiguë du désir de progresser dans un univers, d'explorer et d'élaborer une narration est l'un des points forts des adaptations de Lovecraft dans le genre du jeu vidéo d'aventure (Lessard, 2010, p. 7).

57 Pour que naisse cet intérêt ludique qui est le moteur du parcours narratif, le cybertexte Sunless Sea met en place plusieurs dispositifs : l'implémentation d'aléatoire contrôlé fait abandonner l'idée d'un calcul et d'une recherche d'optimum. Pour maintenir une attitude ludique, il est nécessaire de créer une situation dans laquelle il s'agit de «décider dans l'incertain» (Henriot, 1989, p. 239) en dehors de toute «contrainte absolue » (Henriot, 1989, p. 115).

58 Finalement, le narrateur-joueur de Sunless Sea incarne quasiment le protagoniste lovecraftien par sa curiosité et pas seulement par sa faculté à percevoir passivement (Øystein Slåtten, 2016, p. 67). Il est un acteur engagé dans la résolution d'un problème ; plusieurs éléments du jeu, comme la perpétuelle collecte d'informations sur la partie, agissent comme une récompense et comme une motivation (Medler, 2011). L'œuvre de Lovecraft permet ainsi le développement d'une structure ludique suscitant son engagement.

\section{Conclusion}

La difficulté à replacer un jeu dans sa filiation avec une œuvre littéraire nous a conduit à voir que les remédiations du mythe de Cthulhu pouvaient s'opérer soit de manière 
excessivement littérale, soit au contraire en ne s'appuyant que sur des mécaniques et des conventions de genres vidéoludiques.

La ludoformation comme extension d'une narration par sa mise en monde permet de replacer un jeu dans la filiation d'une œuvre littéraire en contournant ces écueils. Un jeu vidéo peut certes contenir des éléments (objets, personnages, lieux) issus d'une œuvre littéraire, et l'on peut mesurer la fidélité de leur implémentation. Ce monde ludique fonctionne également selon des règles qui fondent une jouabilité définie, règles qui sont quant à elles générales et abstraites, et qui ne permettent de rattacher un jeu qu'à un "genre » aux côtés d'autres systèmes de règles. A la différence de ces lectures, considérer un jeu vidéo comme ludoformation, c'est se placer dans la perspective selon laquelle éléments implémentés et règles générales fonctionnent ensemble, et qu'ils s'informent l'un l'autre. Chaque élément visuel, dispositif de narration ou nom propre a pour fonction de renseigner sur les règles qui régissent l'univers dans lequel elles se trouvent, parfois avec ambiguïté.

61 Sunless Sea peut ainsi être considéré comme une transposition du mythe de Cthulhu du point de vue d'un parcours narratif du protagoniste, mis en parallèle avec celui du joueur. La mise en place d'une narration vidéoludique amène donc la création d'un espace virtuel, mimétique de la circulation du lecteur dans l'espace littéraire. Nous avons vu comment ce jeu propose une expérience de narration hypertextuelle et cybertextuelle d'une coloration spécifique, et avons proposé d'étudier Sunless sea comme une remédiation cybertextuelle. En proposant une expérience de narration hyper et cybertextuelle spécifique, Sunless Sea se révèle en tant que système qui cherche à susciter l'engagement par sa conception de niveau (level design). L'histoire en tant que telle est virtuelle, et "ergodique ": elle n'existe pas avant que l'opérateur (le joueur) n'ait actualisé l'une des issues possibles de cette histoire (Aarseth, 1997, p. 112), issues qui dépendent directement de son expérience de jeu. Deux éléments produisent alors un effet de clôture à la partie du joueur. Tout d'abord la mort, rencontrée fréquemment lors de son parcours, et qui a pour conséquences un retour au port de départ, une perte d'informations (emplacement des îles, dialogues avec les personnages), de marqueurs de progression (quêtes, argent, marchandises et objets précieux), avec la seule possibilité laissée de conserver une partie de cet avancement pour le léguer au prochain protagoniste que le joueur sera amené à incarner. Le second effet de clôture, plus définitif, est celui qui accompagne l'achèvement d'une partie. L'objectif fixé en début de partie est atteint, est sanctionné par l'apparition de l'évènement " a joyous conclusion " [une conclusion joyeuse] et il est possible de créer un nouveau personnage.

C'est ainsi que la traduction vidéoludique d'une œuvre littéraire ne peut se comprendre par sa seule inscription générique, comme le montre le paradigme du cybertexte. Sunless Sea devient alors un texte ludique et narratif, une structure formelle d'actualisation du mythe en réconciliant la présence d'un auteur, qui prévoit un certain nombre d'éléments en plus des règles régissant leur apparition et leur configuration, avec celle du joueur, qui actualise ce qui n'était qu'une potentialité narrative. Une telle compréhension émerge lorsque l'on ne cherche plus seulement le mythe lovecraftien dans les jeux, mais la façon dont le jeu (la stratégie, la résolution de problèmes) peut se trouver à l'origine dans d'autres oeuvres (Zarzycki, 2016, p. 205-206).

Si le mythe de Cthulhu, univers de fiction littéraire élaboré par Lovecraft, est compris comme une transcription de sa façon de voir le monde (Crowley, 2015), l'on constate qu'un récit littéraire est bel et bien « un outil matériel (un moyen) pour donner une réelle 
fondation, stable et relativement immuable, à une œuvre littéraire et ainsi en fournir l'accès au lecteur » (Ingarden, 1973, p. 173). Ludoformer, ce peut être tenter d'effectuer un chemin inverse, d'étendre la présentation d'un fil d'évènements réalisés en un monde ludique, se basant sur le mythe comme sur un monde transmédial (Klastrup, Tosca, 2004, p. 1) : Lovecraft fonde des règles laissées à l'appréciation de ses continuateurs, auteurs, concepteurs, lecteurs ou joueurs.

Comprise dans les coordonnées de la conception de jeu, il est possible de voir comment la proposition soumise par le jeu fait écho à celle de H.P. Lovecraft ; ce dernier présente au lecteur un univers dans lequel le langage, qu'il soit celui de l'auteur ou du protagoniste décrivant l'horreur cosmique qu'il croit percevoir ne suffit pas pour créer du sens (Menegaldo, 2002, p. 372-373). Dérouler une narration consiste pour le joueur à jouer. En amont, pour l'auteur, il s'agit d'écrire dans la langue du possible plutôt que celle du réalisé, à construire et programmer " une machine à produire des mondes possibles " (Eco, 1985, p. 226) opérée par le joueur, qui devient comme on l'a déjà souligné (Campion, Fastrez, 2008) le co-narrateur de ce récit.

\section{BIBLIOGRAPHIE}

AARSETH E. (1997), Cybertext: Perspectives on Ergodic Literature, Baltimore, Johns Hopkins University Press.

AARSETH E. (2001), « Allegories of Space. The Question of Spatiality in Computer Games ». Zeitschrift fur Semiotik, vol. 3-4, n²3, pp. 152-171.

AARSETH E. (2003), « Nonlinearity and Literary Theory », in N. Wardrip-Fruin \& N. Monfort (dir.) , The New Media Reader, Cambridge, The MIT Press, pp. 762-780.

BARNABÉ F. (2014), Narration et jeu vidéo : Pour une exploration des univers fictionnels, Liège, Bebooks. BERRUTI M. (2004), « HP Lovercraft and the anatomy of the nothingness: The Cthulhu Mythos », Semiotica, vol. 150, pp. 363-418.

BLOCH R. (1987), The best of H.P. Lovecraft: bloodcurdling tales of horror and the macabre (1st edition), New York, Ballantine Pub Group.

BOLTER J. D. \& GRUSIN R. (2003), Remediation: understanding new media, Cambridge, MIT Press.

BOUCHARDON S. (2012), « Du récit hypertextuel au récit interactif », Revue de la Bibiothèque nationale de France, $\mathrm{n}^{\circ} 42$, pp. 13-20.

BRATOSIN S. (2007), La concertation dans le paradigme du mythe: de la pratique au sens, Berne, Peter Lang.

BREMOND C. (1966), « La logique des possibles narratifs », Communications, vol. 8, n 1, pp. 60-76. DOI 10.3406/comm.1966.1115.

CAMPION B. \& FASTREZ P. (2008), « Attitude spectatorielle et action dans les jeux vidéo », Recherches en communication, $\mathrm{n}^{\circ} 129, \mathrm{pp} .37-57$. 
CHAPMAN A. (2016), Digital Games as History: How Videogames Represent the Past and Offer Access to Historical Practice, New York, Routledge.

CROWLEY D. A. (2015), «The Arcane and The Rational: Lovecraft's Development of a Unique Mythos ", Discussions vol. 11, n 1, http://www.inquiriesjournal.com/articles/985/the-arcaneand-the-rational-lovecrafts-development-of-a-unique-mythos.

DOR S. (2016), « Rejouer le récit dans différentes adaptations ludiques de l'univers de J.R.R. Tolkien », Sciences du jeu n ${ }^{\circ}$ 5, http://journals.openedition.org/sdj/575

ECO U. (1985), Lector in fabula : ou la coopération interprétative dans les textes narratifs, Paris, Grasset.

FEST, B. (2016). « Metaproceduralism : the Stanley Parable and the legacies of postmodern metafiction ", Wide Screen, vol. 6, $n^{\circ}$ 1, http://www.widescreenjournal.org/index.php/journal/ article/view/105

FLANAGAN K. M. (2017), « Videogame Adaptation », in T. M. Leitch. (dir.), The Oxford Handbook of Adaptation Studies, Oxford University Press, pp. 441-456.

GENETTE G. (1982), Palimpsestes : la littérature au second degré, Paris, Seuil.

GENVO S. (2005a), « Le game design de jeux vidéo : quel type de narration ? », http:// www.archimuse.com/publishing/ichim05/SebastienGenvo.pdf

GENVO S. (2005b), « Transmédialité de la narration vidéoludique : quels outils d'analyse ?» Compar(a)ison, $\mathrm{n}^{\circ}$ 2, pp. 103-112.

HARMS D. (2008), The Cthulhu Mythos Encyclopedia: A Guide to H. P. Lovecraft's Universe, $3^{\text {rd }}$ Revised edition, Lake Orion, Elder Signs Press.

HARVEY M. (2002), « Traduire l'intraduisible. Stratégies d'équivalence dans la traduction juridique », ILCEA, n³. https://journals.openedition.org/ilcea/pdf/790

HENRIOT J. (1989), Sous couleur de jouer : La métaphore ludique, Paris, José Corti.

HITE K. (2007), « Narrative Structure and Creative Tension in Call of Cthulhu », Electronic Book Review. http://www.electronicbookreview.com/thread/firstperson/adventurous.

HOUELLEBECQ M. (2010), H. P. Lovecraft. Contre le monde, contre la vie, Paris, J’ai lu.

INGARDEN R. (1973), The cognition of the literary work of art, Evanston, Northwestern University Press.

JENKINS H. (2004), « Game Design as Narrative Architecture », in P. Harrigan \& N. Wardrip-Fruin (eds.), First Person: New Media as Story, Performance, and Game, Cambridge, The MIT Press, pp. 118-130.

JUUL J. (2005), Half-Real: Video Games Between Real Rules and Fictional Worlds, First Edition, Cambridge, The MIT Press.

KEOGH B. (2014), « Across Worlds and Bodies: Criticism in the Age of Video Games », Journal of Games Criticism, vol. 1, n 1, http://gamescriticism.org/articles/keogh-1-1/.

KLASTRUP L. \& TOSCA S. (2004), « Transmedial Worlds - Rethinking Cyberworld Design », 2013 International Conference on Cyberworlds, Los Alamitos, CA, USA, IEEE Computer Society, pp. 409-416.

KOSTER R. (2005), A theory of fun for game design, Scottsdale, Paraglyph Press.

KRZYWINSKA T. (2009), « Reanimating H. P. Lovecraft », in B. Perron (dir.), Horror Video Game : Essays on the Fusion of Fear and Play, Jefferson, McFarland \& Co Inc, pp. 267-288. 
LESSARD J. (2010), « Lovecraft, le jeu d'aventure et la peur cosmique », Loading... vol. $4, \mathrm{n}^{\circ} 6$. http://journals.sfu.ca/loading/index.php/loading/article/view/89.

LOVECRAFT H. P. (direction et préface par F. LACASSIN) (2010), CEuvres, tome 1, Paris, R. Lafont.

LOVECRAFT H. P. (2013 [1928]) L'Appel de Cthulhu, Paris, Pocket.

MARTI M. (2014), « La narrativité vidéoludique : une question narratologique », Cahiers de Narratologie. Analyse et théorie narratives, $\mathrm{n}^{\circ}$ 27, http://journals.openedition.org/narratologie/7009

MEDLER B. (2011), « Player Dossiers: Analyzing Gameplay Data as a Reward », Game Studies, vol. 11, $\mathrm{n}^{\circ}$ 1. http://gamestudies.org/1101/articles/medler.

MENEGALDO G. (2002), H.P. Lovecraft : fantastique, mythe et modernité, Paris, Editions Dervy.

MITCHELL A. \& MCGEE K. (2009), « Designing storytelling games that encourage narrative play », Joint International Conference on Interactive Digital Storytelling, Berlin, Springer, p. 98-108.

ØYSTEIN SLÅTTEN K. (2016), Humans in a Hostile Cosmos: Science, Cosmicism and Race in HP Lovecraft's Cthulhu Mythos, Mémoire de maîtrise, University of Stavanger. https://brage.bibsys.no/xmlui/ handle/11250/2400445.

PRICE R. M. (1990), H.P. Lovecraft and the Cthulhu mythos, Mercer Island, Starmont House.

RANDALL N. (2017), « Source as Paratext: Videogame Adaptations and the Question of Fidelity », in C. R. Miller ILLER \& A. R. KELLY (eds.), Emerging Genres in New Media Environment Cham, Springer International Publishing, pp. 171-185.

ROBINSON C. L. (2010), « Teratonymy: The Weird and Monstrous Names of HP Lovecraft », Names, vol. 58, n³ 3, pp. 127_138. DOI 10.1179/002777310X12759861710420.

SICART M. (2008), « Defining Game Mechanics », Game Studies, vol. 8, n² 2. http:// gamestudies.org/0802/articles/sicart.

SMITH P. (2011), « H. P. Lovecraft: Re-visioning Romantic-Era Gothicism », Literature Compass, vol. $8, \mathrm{n}^{\circ} 11, \mathrm{pp} .830-839$.

SOLINSKI B. (2017), « A la marge de la lecture et du ludique : les livres-jeux », Sciences du jeu, $\mathrm{n}^{\circ} 7$. http://journals.openedition.org/sdj/777.

ZARZYCKI A. (2016), « Epic video games: Narrative spaces and engaged lives », International Journal of Architectural Computing, vol. 14, n 3, pp. 201-211.

\section{NOTES}

1. La revue Lovecraft Studies, devenue Lovecraft Annual, éditée par S.T Joshi en est un bon exemple.

2. Le jeu figure en deuxième place dans un classement des «meilleurs jeux lovecraftiens" https://solutions.softonic.com/what-are-the-best-lovecraft-based-games-on-steam, consulté le 05/03/17. Sur plusieurs plateformes de discussion telles que Reddit, les forums de CanardPC ou encore la plateforme de jeux Steam, les joueurs discutent de la présence d'éléments de l'univers lovecraftien dans le Sunless Sea, https://steamcommunity.com/app/304650/ discussions/0/341537388317802084/, consulté le 06/03/17.

3. H.P. Lovecraft reconnaît « [qu'] il n'est pas possible de créer un objet qui dégage une horreur même dix fois moindre que celle qu'il est sensé suggérer. Si on tente d'écrire le Nécronomicon, le résultat sera une déception pour tous ceux qui ont tremblé à la lecture des allusions obscures faites à son sujet » (H. P. Lovecraft, in Lacassin, 2010, p. 1049). 
4. Au sens de Bolter et Grusin, comme un «emprunt complexe dans lequel un medium est luimême incorporé ou représenté dans un autre medium » (Bolter, Grusin, 2003, p. 45).

5. L'origine du terme renvoie à une vision de la conception de jeu vidéo (video game design) qui est celle de la construction d'un espace ou d'un environnement virtuel. Ce terme que nous proposons se veut la traduction du processus qu'Espen Aarseth nomme ludoforming. Ce dernier l'emploie pour la première fois lors de l'évènement «Ludotopia » qui s'est tenu en 2010 à Copenhague. Il s'agit de désigner la transposition d'espaces, réels ou fictifs, en espaces de jeu. Dans le cas du jeu vidéo, cela se caractérise notamment par une anticipation des possibles interactions du joueur avec l'environnement et la création d'affordances ludiques.

6. Défini comme « toute relation unissant un texte B [...] à un texte antérieur A [...] sur lequel il se greffe d'une manière qui n'est pas celle du commentaire» (Genette, 1982, p. 13).

7. https://lovecraftzine.com/lovecraftian-video-games/ consulté le 31/03/16. Le Lovecraft Ezine est un magazine en ligne consacré à Lovecraft et plus généralement au genre de la weird fiction, à l'horreur cosmique et au mythe de Cthulhu.

8. http://www.hplovecraft.com/popcult/games/digital.aspx, consulté le 01/04/16.

9. https://en.wikipedia.org/wiki/Category :Video_games_based_on_works_by_H._P._Lovecraft, consulté le 31/03/16

10. Ce type de mécaniques se déploie notamment dans le jeu The Stanley Parable (2013), un jeu dont Bradley Fest propose de dire qu'il fonctionne sur un mode «métaprocéduraliste » : le jeu met en place des processus (par les règles du jeu) qui expriment la vacuité d'autres processus (les règles sociales, les injonctions hiérarchiques) (Fest, 2016).

11. Le brouillard de guerre (ou « fog of war ») désigne le manque d'information des combattants et commandants durant les combats (unités ennemies non visibles, identification incertaine des unités découvertes, etc.

12. «Ce qui compte est le jeu [the play of the game], avec tout ce qu'il réclame en termes de competence technique et mécanique. La source et l'histoire ne sont que paratexte.» (Randall, 2017, p. 184).

\section{RÉSUMÉS}

Selon Lovecraft, toute tentative de matérialisation trop explicite de l'horreur, suggérée dans son œuvre, ne saurait que lui nuire. Quelles sont alors les spécificités propres au support vidéoludique convoquées pour cadrer l'expérience de jeu et tenir le pari de l'effroi et de l'irreprésentable? Cet article propose d'étudier le jeu vidéo Sunless sea comme une «ludoformation» du mythe de Cthulhu: une remédiation qui cherche à maintenir une forme d'équivalence et de continuité sémantique entre une source et son actualisation vidéoludique. $\mathrm{Au}$ moyen d'une analyse de la conception de niveau (level design) et de l'architecture narrative que ce jeu met en place, il s'agira de voir comment la proposition soumise par le jeu permet de redéployer une forme de narration lovecraftienne. Ce paradigme de la ludoformation souhaite donner de nouvelles perspectives sur le lien d'analogie entre œuvre littéraire et jeu vidéo : voir le mythe de Cthulhu comme une matrice de laquelle émergent des univers fictionnels. La transposition en jeu vidéo repose ici sur une remédiation qui prend la forme d'une mise en monde. 
According to Lovecraft, any attempt to explicitly materialize the suggested horror within his works could only do harm to it. What are the videogame's characteristics that are invoked to frame the ludic experience and address the challenge of the horror and the unrepresentable? This article proposes to examine the videogame Sunless Sea as a "ludoformation" of the Cthulhu mythos: a remediation seeking to maintain a form of equivalence and semantic continuity between a source and its videogame realization. By means of an analysis of its level design and the narrative architecture implemented by the game, the goal is to look at the game's proposition as a way to reenact a kind of lovecraftian narration. This ludoformation paradigm wishes to give new perspectives regarding the analogy that can link literary work and videogames : looking at the Cthulhu mythos as a matrix from which emerge fictional universes. The transposition into videogames is based here on a remediation which takes the form of worldbuilding.

INDEX

Mots-clés : Lovecraft, Sunless Sea, cybertexte, ludoformation, transposition, narration, exploration

Keywords : Lovecraft, Sunless Sea, cybertext, ludoformation, transposition, narration, exploration

\section{AUTEUR}

\section{JULIEN BAZILE}

Université de Lorraine / Université de Sherbrooke 